\title{
Surgical Case Deferment During a Global Pandemic
}

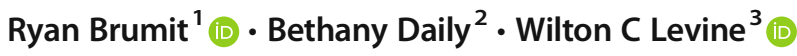

Received: 24 April 2020 / Accepted: 7 May 2020/Published online: 3 June 2020

(C) Springer Science+Business Media, LLC, part of Springer Nature 2020

In March of 2020, hospitals across the United States began deferring elective surgical procedures in response to the rapidly escalating SARS-CoV2 pandemic. On March 15, 2020, the Massachusetts Department of Public Health (DPH) issued a directive that all non-essential surgery be deferred [1]. Massachusetts General Hospital (MGH) needed to quickly evolve surgical scheduling guidelines that were clear, reasonable and fair to meet the needs of our patients and balance the evolving needs of our hospital and healthcare system. On March 17, 2020, the American College of Surgery (ACS) published guidance for triage of non-emergent surgery [2]. A method was needed to quickly draw-down our operating room $(\mathrm{OR})$ schedule to be in line with the ACS guidelines and more detailed guidelines that we developed. This was necessary given the rising need to conserve space, staffing and PPE resources. We describe a method for case deferment that empowers a large swath of surgical specialties to make safe and quick decisions that result in a dramatic reduction of the OR schedule and hospital census. The following rules and methodology have applicability for our current pandemic as well as during other emergency situations such as blizzards, hurricanes or massive power outage.

\section{Stakeholders and Scope}

Surgical and Perioperative leadership determined a set of focused criteria to guide types of patient care deemed essential

This article is part of the Topical Collection on Patient Facing Systems

Ryan Brumit

rbrumit@partners.org

1 Perioperative Services, Massachusetts General Hospital Boston, Boston, MA, USA

2 Perioperative Service and Healthcare Systems Engineering, Massachusetts General Hospital, Boston, MA, USA

3 Perioperative Services and Medical Procedure Areas, Massachusetts General Hospital, Boston, MA, USA during the global pandemic and during the DPH mandate to defer all non-essential surgery. MGH evolved the ACS guidelines to specifically include the following patient/disease states to have ongoing surgery:

1. Life and limb saving procedures

2. Treatment of life threating / unstable disease

3. Treatment of infections that would otherwise become life threatening

4. Cancer

a. Cancers in patients who have completed their neoadjuvant therapy and are in the window of resectability, and for whom non-operative temporizing maneuvers are not possible

b. Aggressive cancers that will grow significantly in 2 months for which other therapies cannot be used to temporize (e.g. triple-negative breast cancer)

c. Second part of staged procedures in which the first stage has been completed (e.g. patient has an open wound awaiting reconstruction)

d. Diagnostic procedure required to allow initiation of appropriate cancer therapy (e.g. diagnosis of lymphoma or diagnosis of metastatic cancer)

5. Acute symptoms (e.g. GI bleeding, bowel obstruction, dysphagia and/or aspiration risk, airway encroachment) for which alternative therapy is not appropriate

6. Fractures needing surgical repair

Implementation of effective surgical case deferral requires a multidisciplinary team. MGH has leveraged the following role groups to adhere to its case deferment directive:

- Perioperative Services Administration: development and facilitation of method steps

- Medical Director: ownership of the method and management of the OR schedule

- Surgical Chiefs: ownership of deferment decisions and non-deferrable case approval 
- Surgeons/Surgical Offices: deferred patient communication

- Central Scheduling: Electronic Health Record (EHR) processing of all deferrable cases

- IS/EHR Resources: development and distribution of standard reporting tools

Beyond those directly involved in the process of deferring surgical cases, Nursing and Anesthesia must be kept well informed of significant drops in OR volume, as their staff are crucial in times of crisis and will be needed for redeployment to support other critical care needs within the hospital. Beyond the operating room, MGH used this method to defer scheduled cases in all procedural areas including endoscopy, electrophysiology, cardiac cath and interventional radiology.

\section{Initial Framework}

The framework of this method starts with generation of a report containing upcoming OR cases. Key data points include: Date of Surgery, Patient Name, Service, Surgeon, Procedures, Pre-Op Diagnosis, and patient contact information. Decisions must then be made on which cases can be deferred.

A set of 12 strategically ordered update queries was created to provide rudimentary deferability recommendations based on keywords found in the procedure booking code and pre-op diagnosis (fig. 1a). In the first 2 weeks, 1000 scheduled cases evaluated by these queries. $67 \%$ of them were determined by the logic and automatically marked with a deferability recommendation of high (easy to defer), medium or low (case must proceed). For example, the word arthroplasty automatically establishes a high deferability recommendation, whereas the words malignant or cancer automatically establish a low deferability recommendation. Based on the order of the update queries, a low deferability cancer case converts to medium deferability if the word prostatectomy is found. Inpatients are always automatically marked as not deferrable.

As to not get too far ahead of the pandemic's trajectory, 2 weeks of upcoming scheduled case data is exported from the EHR every 2 weeks and run through the query logic. After generating the initial recommendations, the file is then divided into service-specific case lists and distributed to Chiefs and Administrators for formal review. Instructions are to 1) indicate which cases can be deferred and resubmit the updated file within $24 \mathrm{~h}$, and 2) contact all deferred patients to let them know that their surgery has been postponed within $48 \mathrm{~h}$.

\section{Electronic Health Record Deferment Process}

Rather than canceling en masse, deferrable cases are removed from the OR schedule grid and placed back into a scheduling depot to make rescheduling easier when the hospital returns to normal operations. A far future date was selected as the new "date of surgery" for all cases being deferred. In our case, we chose $1 / 1 / 2022$, so that that cases can be easily tracked and isolated from any non-deferrable cases (fig. 1b). The work of processing all deferred cases has been centralized in order to maintain consistency and ensure that all deferrable cases are removed from the schedule in a timely and organized manner.

\section{Focusing the Schedule}

With a significant reduction in surgical volume, the need to decrease the number of daily rooms running became quickly apparent. 58 blocked operating rooms open and available on the OR scheduling grid meant there was no visual guidance for where non-deferrable cases should be placed. This created unnecessary work for OR leaders and the central scheduling team, as they had to actively consolidate rooms and surgical services throughout the day to effectively manage operating room resources. To rectify this issue, the OR schedule was reduced first to 25 blocked rooms per day and over subsequent weeks ultimately down to 10 blocked rooms per day for both scheduled necessary surgery as well as urgent/emergent surgery. Our normal OR schedule is mostly comprised of blocks assigned to specific surgeons, which was simply not practical to maintain at this time. The reallocation of blocks to servicelevel assignments was based upon a service's anticipated nondeferrable case volume.

Within our EHR, the normal OR block schedule recurs indefinitely. When permanent block changes are made, the new assignment overwrites the old by beginning on a specific date and recurring indefinitely. As case deferment occurs in two-week windows, the temporary block schedule can be set in the EHR to coincide, protecting the normal block schedule from being deleted.

\section{Improving the Framework}

An improvement to the framework was the inclusion of a formal process for scheduling any new non-deferrable cases. Knowing that certain patients need their surgeries to occur before the end of the crisis is reached, all new nondeferrable cases must go through a service-based review carried out by the Surgical Chief (or designee) prior to scheduling. A schedule change report was also created so that the OR Medical Director can conduct a daily review of all new cases that have been added to the schedule and follow-up with individual surgeons and surgical leadership as needed.

It was also found that the Pre-Procedure Evaluation (PPE) program had fallen out of synch with the OR because the twoweek cadence of the deferment method did not align with the 30 -day scheduling window of the pre-procedure phone 
Fig. 1 Overview of the MGH surgical case deferment method and its impact on operating room volume. a: Application of Rule for Deferment Recommendation. b: Process Map of Surgical Case Deferment Method. c: Surgical Case Volume \& Deferment - BiWeekly Trend
Application of Rules for Deferment Recommendation Single-Case Example

a

Service: Urology

Procedure: Prostatectomy

Pre-Op Diagnosis: Prostate Cancer

Deferment Recommendation (based on queries run below): Medium
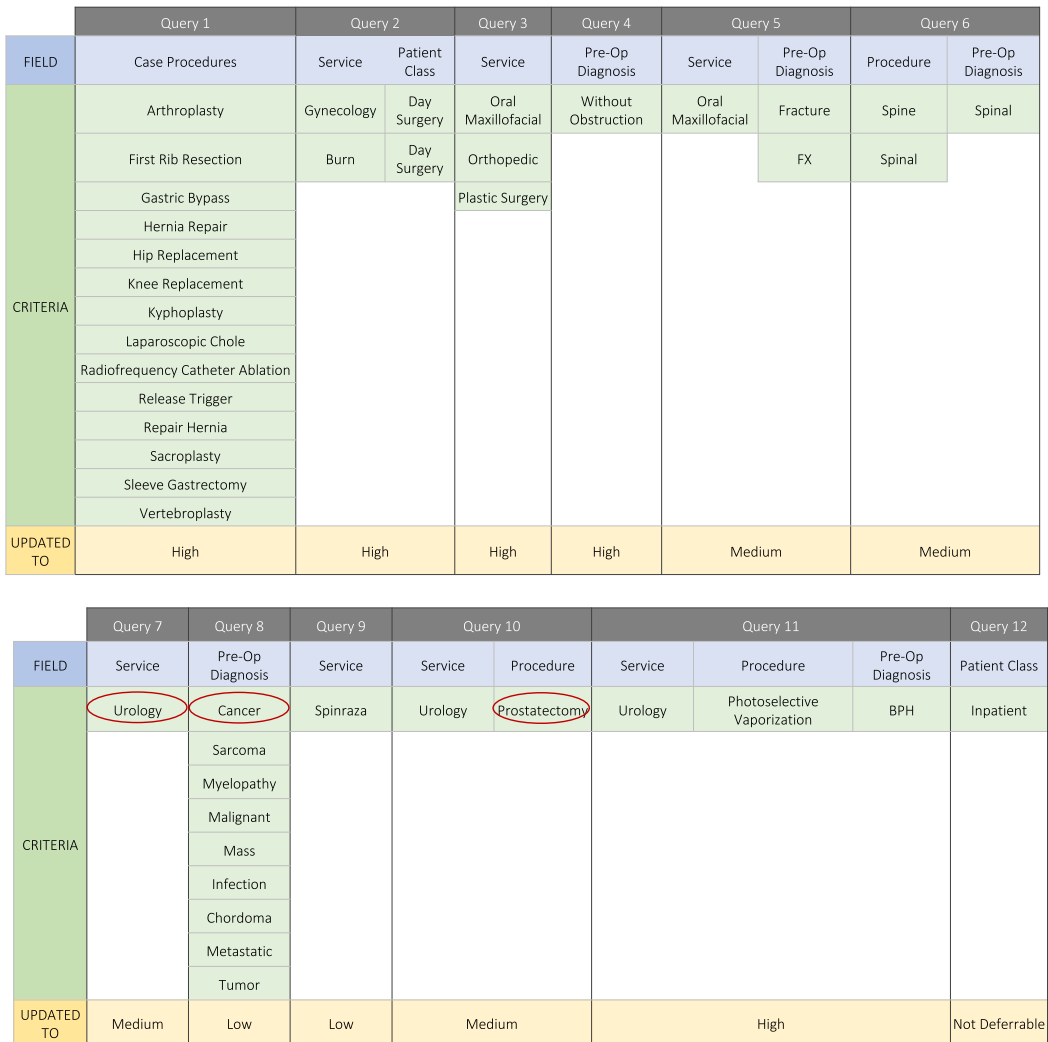

b

Process Map of Surgical Case Deferment Method

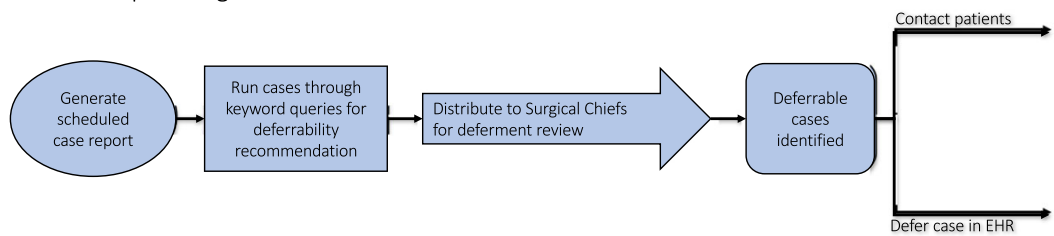

C

Surgical Case Volume \& Deferment - Bi-Weekly Trend

1600

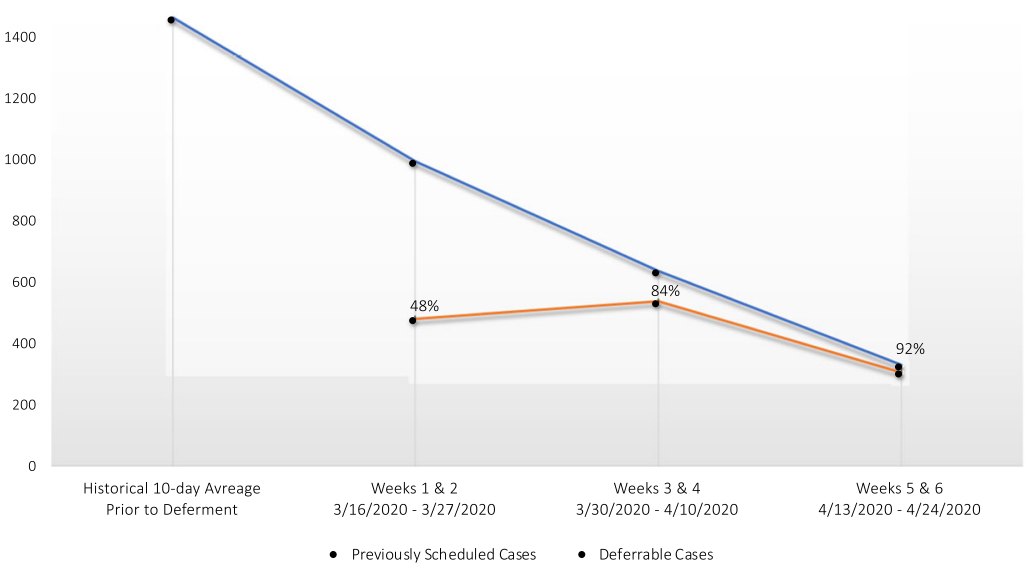


interviews. Nurses were calling patients and conducting interviews for noticeably deferrable cases, simply because they were scheduled for a date of surgery beyond the two-week deferment window. To address this, an OR Schedule Report was built for the phone nurses, which is populated by surgical cases scheduled over the next five business days and ignores the PPE appointment date and time, ensuring that the phone nurses are only calling non-deferrable patients.

\section{Summary}

As of 4/6/2020, MGH's surgical case deferment method has been employed for three two-week windows. In this time, the deferment rate has dropped from $48 \%$ (weeks $1 \& 2$ ) to $92 \%$, leaving only 25 non-deferrable cases on the schedule across 10 days (weeks $5 \& 6$ ) (fig. 1c).

Considerable curtailing of OR volume has had a significant positive impact on hospital operations. Limiting the flow of OR patients to the ICUs and inpatient floors increases the available capacity for Covid-positive and Covid-risk patients and provides flexibility with Covid floor consolidation. Reduction in our OR schedule has allowed re-deployment of our nursing and anesthesia teams to help staff and support new ICUs that have opened and allowed our surgeons to help staff emerging respiratory clinics. Decreasing the staff and PPE required for the operating room has helped our hospital to remain stable during these uncertain times.

\section{Compliance with Ethical Standards}

Funding No funding was received in support of this work.

Conflict of Interest Mr. Ryan Brumit declares that he has no conflict of interest. Mrs. Bethany Daily declares that she has no conflict of interest. Dr. Wilton C Levine declares that he has no conflict of interest.

Ethical Approval This article does not contain any studies with human participants or animals performed by any of the authors.

Informed Consent N/A

\section{References}

1. Baker C. Elective Procedure Order [Mass.gov web site]. March 15, 2020. Available at: https://www.mass.gov/doc/march-15-2020elective-procedures-order. Accessed on: April 5, 2020.

2. COVID-19: Guidance for Triage of Non-Emergent Surgical Procedures [American College of Surgeons web site]. March 17, 2020. Available at: https://www.facs.org/covid-19/clinicalguidance/triage. Accessed on: April 9, 2020.

Publisher's Note Springer Nature remains neutral with regard to jurisdictional claims in published maps and institutional affiliations. 